\title{
Modulation of Baroreflex Function by Rosiglitazone in Prediabetic Hyperglycemic Rats
}

\author{
L.-Z. HONG ${ }^{1,2}$, Y.-C. CHAN ${ }^{2}$, M.-F. WANG ${ }^{2,3}$, J.-Y. WANG ${ }^{4}$, S.-W. HUNG ${ }^{5}$, C.-I. TSAI ${ }^{6}$, \\ C.-J. TSENG ${ }^{7}$ \\ ${ }^{1}$ Department of Medical Education and Research, Taichung Veterans General Hospital, Taichung, \\ Taiwan, ${ }^{2}$ Department of Food and Nutrition, Providence University, Taichung, Taiwan, \\ ${ }^{3}$ Department of Food Science, Yuanpei University, HsinChu, Taiwan, ${ }^{4}$ Basic Medical Science, \\ Department of Nursing, Hung-Kuang University, Taichung, Taiwan, ${ }^{5}$ Department of Radiology, \\ Taichung Veterans General Hospital, Taichung, Taiwan, ${ }^{6}$ Department of Traditional Chinese \\ Medicine, Taichung Veterans General Hospital, Taichung, Taiwan, ${ }^{7}$ Department of Medical \\ Education and Research, Kaohsiung Veterans General Hospital, Kaohsiung, Taiwan
}

Received December 14, 2011

Accepted May 22, 2012

On-line August 8, 2012

\section{Summary}

Baroreflex sensitivity (BRS) is abnormal in the prediabetic state. This study was conducted to determine effects of chronic rosiglitazone (RSG), an insulin sensitizer, on BRS in prediabetic hyperglycemic $(\mathrm{PDH})$ rats induced by nicotinamide and streptozotocin. The fasting and postprandial blood glucose levels were 5.6-6.9 and 7.8-11.0 mmol/l, respectively. Rats were treated with RSG or saline for 12 weeks. BRS response to phenylephrine (PE-BRS) or sodium nitroprusside (NP-BRS) was determined by linear regression method. Cardiac sympathetic and parasympathetic influences were determined by autonomic blockades. In the saline-treated PDH rats, PE-BRS was enhanced early at week 4 and became greater at week 12 . Abnormalities in NP-BRS and cardiac autonomic influences were found only after week 12. Four weeks of RSG treatment normalized blood glucose levels but not PE-BRS. All altered cardiovascular variables were completely restored by 12 weeks of RSG treatment. The correlation between BRS and blood glucose levels in salinetreated PDH rats was significant at week 12, but no correlation was found in RSG-treated rats. In conclusion, hyperglycemia, even in the prediabetic state, may play a role in BRS abnormalities. RSG treatment early in the prediabetic state may normalize BRS via cardiac autonomic modulation, besides its antihyperglycemic action.

\section{Key words}

Autonomic function - Baroreflex • Diabetes - Hyperglycemia • Rosiglitazone

\section{Corresponding authors}

Ling-Zong Hong, Department of Medical Education and Research, Taichung Veterans General Hospital, Taichung, Taiwan, 160 Section 3 Taichung-Kang Road, Taichung, 40705, Taiwan, ROC. Fax: +886-4-2359-2705. E-mail: Izhong@vghtc.gov.tw and

Ching-Jiunn Tseng, Department of Medical Education and Research, Kaohsiung Veterans General Hospital, 386-Ta Chung 1st Road, Kaohsiung, Taiwan, ROC. Fax: +886-7-346-8056. E-mail: cjtseng@vghks.gov.tw

\section{Introduction}

Autonomic dysfunction may occur early in the progression of diabetes and is an important risk factor in predicting cardiac morbidity and mortality in diabetic patients (Vinik et al. 2003). Arterial baroreflex sensitivity (BRS), an index for evaluating cardiac autonomic function, is known to be impaired in prediabetes (Iellamo et al. 2006) and diabetes (Dalla-Pozza et al. 2007, Maeda et al. 1995). Hyperglycemia has been implicated as a pathogenic factor for diabetic autonomic dysfunction (Stein et al. 2007, Wu et al. 2007). BRS has also been 
shown to be significantly reduced in response to acute hyperglycemia (Marfella et al. 2001) and an inversed relationship has been found to exist between BRS and blood glucose levels (Lefrandt et al. 2000) in healthy subjects.

It has been reported that intensive glycemic control could retard the development of cardiovascular autonomic dysfunction in diabetic patients (The Diabetes Control and Complications Trial Research Group 1998). In addition, insulin intervention immediately following induction of diabetes has been shown to normalize the abnormal BRS in experimental diabetic models with insulin deficiency (Chang and Lund 1986, Parra et al. 2005). Thus, early interventions which improve glycemic control may have beneficial effects on diabetic autonomic dysfunction.

Rosiglitazone (RSG), an insulin sensitizer, is widely used clinically as an anti-hyperglycemic agent in type 2 diabetes because of its effects on glucose and lipid metabolism (Yki-Jarvinen 2004). Besides its insulinsensitizing property, RSG also has benefits on cardiovascular functions, including improvement in endothelial function and lowering of blood pressure (Kelly and Bank 2007). Interestingly, these cardiovascular actions of RSG have also been found in type 1 diabetic animals (Wang et al. 2007). It is not known whether RSG treatment, especially early in the prediabetic state, might favor the glycemic control and baroreflex function.

Therefore, the present study was undertaken to investigate the effects of chronic RSG treatment on BRS abnormality in rats with prediabetic hyperglycemia which was induced by nicotinamide plus streptozotocin. This model has been reported to be able to induce stable moderate hyperglycemia without markedly affecting the metabolic function of $\beta$-cells (Masiello et al. 1998, Novelli et al. 2001).

\section{Materials and Methods}

\section{Animal preparation}

Male Sprague-Dawley rats weighing 200-250 g were purchased from the National Laboratory Animal Center (Taipei, Taiwan). The rats were allowed free access to the regular chow diet and housed in individual cages with a 12:12-h dark-light cycle. All surgical procedures and experimental protocols were conducted according to the recommendations and approval of the Institutional Animal Care and Use Committee of
Taichung Veterans General Hospital, Taichung, Taiwan.

Rats were fasted overnight and administered nicotinamide intraperitoneally $(230 \mathrm{mg} / \mathrm{kg}$, dissolved in $0.9 \%$ saline; Sigma, St. Louis, MO, USA) before an intravenous administration of streptozotocin $(65 \mathrm{mg} / \mathrm{kg}$, dissolved in $0.1 \mathrm{~mol} / 1$ citric acid, $\mathrm{pH}$ 4.5; Sigma) (Masiello et al. 1998). With combined injection of nicotinamide plus streptozotocin, nicotinamide is able to partially prevent the destruction of pancreatic $\beta$-cells (40\% reduction) by the streptozotocin and the residual $\beta$ cells appear to remain well differentiated and maintain most of metabolic function (Novelli et al. 2001). Thus, rats treated with nicotinamide plus streptozotocin could exhibit a stable moderate hyperglycemia, glucose intolerance, altered glucose-stimulated insulin secretion, and responsiveness to tolbutamide that was reported to be similar to human non-insulin-dependent diabetes mellitus (Masiello et al. 1998). According to the criteria of hyperglycemia for diabetic diagnosis (Genuth et al. 2003), one week after the injections, rats with fasting and postprandial blood glucose levels of 5.6-6.9 and 7.8-11.0 $\mathrm{mmol} / \mathrm{l}$, respectively, were used as the prediabetic hyperglycemic group (PDH; $n=72)$. After the development of prediabetic hyperglycemia was confirmed, PDH rats were randomly assigned to two groups treated with saline or RSG (rosiglitazone maleate, $8 \mathrm{mg} / \mathrm{kg}$ per day, p.o.; Avandia, GlaxoSmithKline SB Pharmaco Inc., Cidra, Puerto Rico) for 1, 4, or 12 weeks, respectively (Hsieh and Hong 2008). A group of agematched euglycemic rats without RSG or saline treatment was used as the control group $(n=36)$. Another group of rats, which were treated with intravenous injection of streptozotocin $(65 \mathrm{mg} / \mathrm{kg})$ only and had fasting and postprandial blood glucose levels of $\geq 7.0 \mathrm{mmol} / 1$ and $\geq 13.8 \mathrm{mmol} / 1$, respectively, were used as the diabetic hyperglycemic rats $(\mathrm{DH} ; n=12)$.

Overnight fasting and postprandial $(2 \mathrm{~h}$ after feeding) blood samples were collected from the tail vein between 9:00 and 11:00 A.M. With the exception of immediate blood glucose assays, plasma samples were separated into several aliquots and stored at $-80{ }^{\circ} \mathrm{C}$ for later analysis. Blood glucose and plasma insulin levels were measured before (week 0) and after 1, 4, 8, and 12 weeks of saline or RSG treatments.

At the end of 1, 4 and 12 weeks of saline or RSG treatments, rats were anesthetized by chloral hydrate (400 mg/kg, intraperitoneally; Sigma) prior to implanting vascular catheters (Micro-Renathane tube, MRE 040, $1.02 \mathrm{~mm}$ outer diameter $\times 0.64 \mathrm{~mm}$ inner diameter) in the 
right femoral artery for later blood pressure measurements and blood sampling, and in the left femoral vein (MRE 033, $0.84 \mathrm{~mm}$ outer diameter $\times 0.36 \mathrm{~mm}$ inner diameter) for drug administration. The catheters were filled with heparinized saline $(20 \mathrm{U} / \mathrm{ml})$, exteriorized through the dorsal midscapular region of the animal, and covered with a stainless-steel extension spring. Rats were allowed to recover for a minimum of 5 days. During the recovery period, rats were monitored for signs of infection, body weight gain, behavior, and food and water intakes. Only apparently healthy animals with no signs of pain and infection that were freely and actively moving and gaining weight normally were used for the experiments.

\section{Arterial blood pressure and heart rate recording}

In experiments, the arterial catheter was connected to a pressure transducer (Gould Statham P23Db, Gould Inc., Oxnard, California, USA) fed to a polygraph system (Pressure Processor and TA4000 thermal array recorder, Gould Inc.). The signals were also stored on a tape recorder (Neuro-Corder DR-890, Neuron Data, New York, USA) for later analysis. The rats were left in the experimental cage at least $1 \mathrm{~h}$ before the experiment. Rats were conscious and unrestrained during the experiment. The experimental environment was kept as quiet as possible to avoid any interference in blood pressure or heart rate (HR). The baseline measurements for arterial blood pressure, mean arterial blood pressure (MAP), and HR were recorded for at least $10 \mathrm{~min}$ before drug administration in conscious rats.

\section{Cardiac sympathetic and parasympathetic influences}

Cardiac sympathetic and parasympathetic influences and the intrinsic heart rate (IHR) were evaluated at the end of 1, 4 and 12 weeks of saline or RSG treatments based on the chronotropic effects of methylatropine bromide (muscarinic receptor blocker, $4 \mathrm{mg} / \mathrm{kg}$, i.v.; Sigma) and propranolol ( $\beta$-adrenergic receptor blocker, $5 \mathrm{mg} / \mathrm{kg}$, i.v.; Sigma) as previously described (Hsieh and Hong 2008). The cardiac parasympathetic influence was calculated by the difference between the baseline $\mathrm{HR}$ and the methylatropine-induced HR. The sympathetic influence was calculated by the difference between the baseline HR and the propranolol-induced HR. For comparison, the cardiac parasympathetic and sympathetic influences were expressed as absolute values in the present study. The IHR was defined by the HR after adrenergic and cholinergic receptor blockades with both methylatropine and propranolol. The efficacy of propranolol or methylatropine was determined by elimination of the tachycardic responses to isoproterenol $(0.1$ and $1 \mu \mathrm{g} / \mathrm{kg}$, i.v.; Sigma) or the bradycardic responses to acetylcholine ( 0.1 and $10 \mu \mathrm{g} / \mathrm{kg}$, i.v.; Sigma) by more than $95 \%$.

\section{$B R S$}

Baroreflex function was determined using linear regression by plotting the reflex HR changes against the moderate changes in blood pressure elicited by bolus injections of various doses of phenylephrine (PE; 0.2-10 $\mu \mathrm{g} / \mathrm{kg}$, i.v.; Sigma) or sodium nitroprusside (NP; 0.2-10 $\mu \mathrm{g} / \mathrm{kg}$, i.v.; Sigma) in each rat at the end of 1,4 and 12 weeks of saline or RSG treatments (Hsieh and Hong 2008). Slope of the regression line was used as the index of BRS for each PE or NP test. Values of PE- and NPBRS were calculated separately from each rat in each BRS evaluation. The contributions of sympathetic and parasympathetic components in BRS were further determined by propranolol and methylatropine, respectively.

\section{Biochemical analysis}

The whole-blood glucose levels were measured by the glucose oxidase method with the YSI glucose analyzer (YSI 2300 Plus; Yellow Springs Instruments, Yellow Springs, Ohio, USA). Plasma insulin levels were measured by solid-phase two-site enzyme immunoassay techniques using a commercial rat insulin enzyme-linked immunosorbent assay kit (Mercodia AB, Uppsala, Sweden).

\section{Data analysis}

The experimental results were evaluated by twoway repeated measures analysis of variance (ANOVA). Bonferroni's test was applied for post hoc analysis when ANOVA detected a statistical significance for one of the factors. Correlation of PE-BRS and NP-BRS with fasting and postprandial blood glucose levels were analyzed by Pearson's correlation analysis. $P<0.05$ was considered statistically significant. Values are expressed as means \pm SEM. 

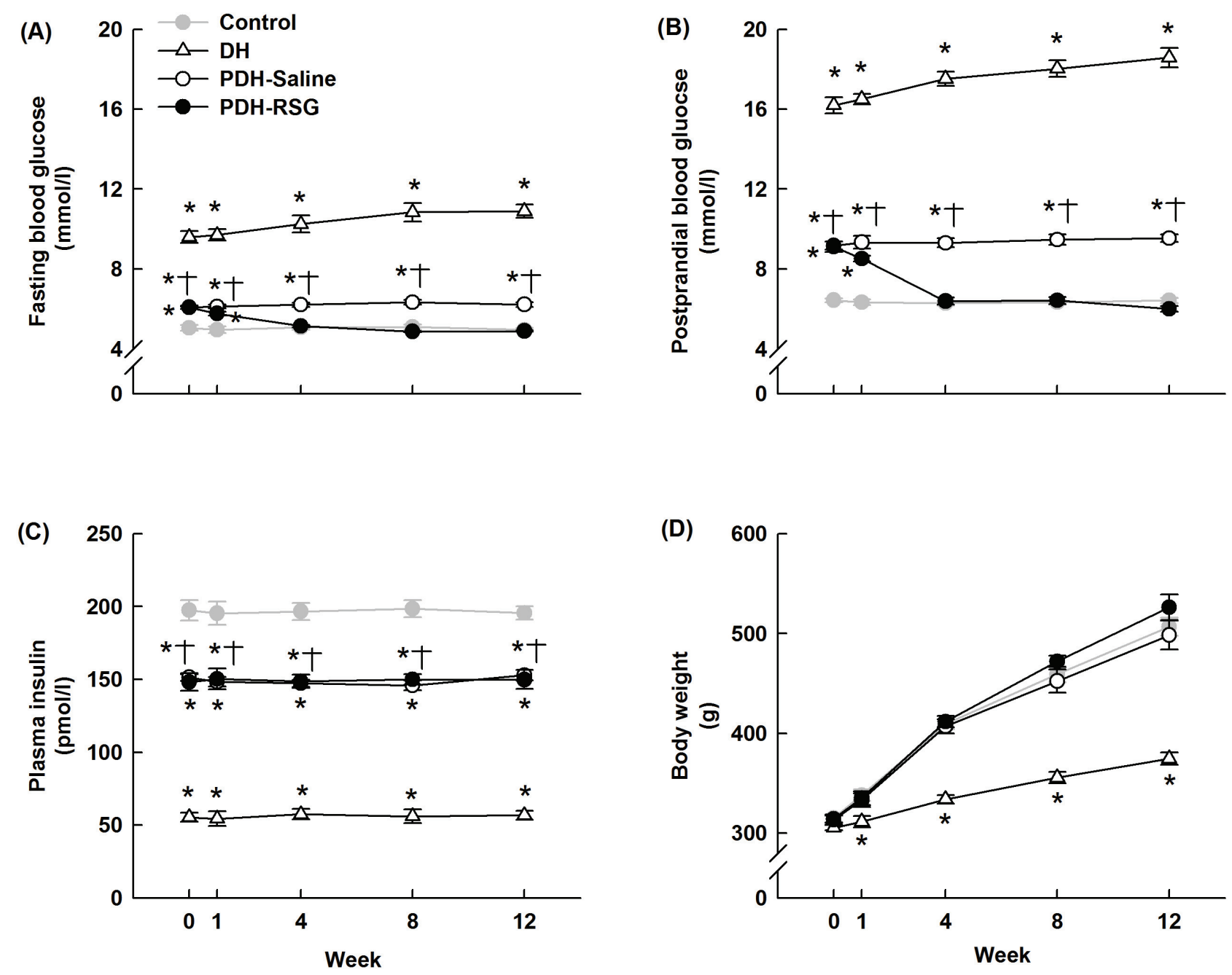

Fig. 1. Blood glucose levels, plasma insulin levels, and body weights in the control, $D H$, and PDH rats during the 12-week period. (A, B) Fasting and postprandial blood glucose levels, (C) plasma insulin levels, and (D) body weights. The PDH rats were subjected to saline or RSG treatments for 12 weeks. $n=12$ per group. $* P<0.05$ vs. the corresponding values of control rats; $\dagger P<0.05$ vs. the corresponding values of $\mathrm{DH}$ rats. Values are expressed as mean $\pm \mathrm{SEM}$.

\section{Results}

Blood glucose levels, plasma insulin levels, and body weights

At week 0 , the fasting and postprandial blood glucose levels were $5.8 \pm 0.1$ and $9.2 \pm 0.2 \mathrm{mmol} / 1$ in the $\mathrm{PDH}$ rats, $4.8 \pm 0.1$ and $6.2 \pm 0.2 \mathrm{mmol} / 1$ in the control rats, and 9.6 \pm 0.3 and $16.2 \pm 0.4 \mathrm{mmol} / \mathrm{l}$ in the $\mathrm{DH}$ rats (Fig. $1 \mathrm{~A}$ and $\mathrm{B})$, respectively. The blood glucose levels of the $\mathrm{PDH}$ rats were in the range of prediabetic state (Genuth et al. 2003). The plasma insulin levels in the $\mathrm{PDH}$ and $\mathrm{DH}$ rats at week 0 were about $80 \%$ and $30 \%$ of the control, respectively (Fig. 1C). The body weights of $\mathrm{PDH}$ rats were not different from those of control rats, while the body weights of $\mathrm{DH}$ rats were significantly lower $(P<0.05)$ (Fig. 1D). The blood glucose and plasma insulin levels in the $\mathrm{PDH}$ rats remained unchanged throughout the 12-week experimental period without RSG treatment.

With one week of RSG treatment, both the fasting and postprandial blood glucose levels in the PDH rats were gradually but incompletely normalized (Fig. 1A and B). However, by the end of week 4, the blood glucose levels were completely restored to the control level and were maintained to the end of week 12. Plasma insulin levels and body weights of the PDH rats were not altered by RSG treatment (Fig. 1C and D).

MAP, HR, IHR, and cardiac sympathetic and parasympathetic influences

At the end of 1,4 , and 12 weeks, the baseline MAP and HR in the PDH rats with or without RSG treatments were not different from those of the timematched control rats (Table 1). 
Table 1. The baseline MAP and HR in the saline- or RSG-treated PDH and control rats at the end of 1,4 and 12 weeks.

\begin{tabular}{|c|c|c|c|c|c|c|c|c|c|}
\hline & \multicolumn{3}{|c|}{ Week 1} & \multicolumn{3}{|c|}{ Week 4} & \multicolumn{3}{|c|}{ Week 12} \\
\hline & \multirow{2}{*}{$\begin{array}{c}\text { Control } \\
(n=12)\end{array}$} & \multicolumn{2}{|c|}{ PDH } & \multirow{2}{*}{$\begin{array}{c}\text { Control } \\
(n=12)\end{array}$} & \multicolumn{2}{|c|}{ PDH } & \multirow{2}{*}{$\begin{array}{c}\text { Control } \\
(n=12)\end{array}$} & \multicolumn{2}{|c|}{ PDH } \\
\hline & & $\begin{array}{l}\text { Saline } \\
(\mathrm{n}=12)\end{array}$ & $\begin{array}{c}\text { RSG } \\
(n=12)\end{array}$ & & $\begin{array}{l}\text { Saline } \\
(\mathrm{n}=12)\end{array}$ & $\begin{array}{c}\text { RSG } \\
(n=12)\end{array}$ & & $\begin{array}{l}\text { Saline } \\
(\mathrm{n}=12)\end{array}$ & $\begin{array}{c}\text { RSG } \\
(n=12)\end{array}$ \\
\hline $\begin{array}{l}\text { MAP } \\
(m m H g)\end{array}$ & $115 \pm 5$ & $115 \pm 4$ & $114 \pm 3$ & $116 \pm 4$ & $115 \pm 3$ & $115 \pm 3$ & $114 \pm 4$ & $113 \pm 3$ & $115 \pm 3$ \\
\hline$H R(b p m)$ & $345 \pm 3$ & $344 \pm 2$ & $345 \pm 4$ & $345 \pm 4$ & $345 \pm 5$ & $346 \pm 4$ & $352 \pm 3$ & $353 \pm 2$ & $351 \pm 2$ \\
\hline
\end{tabular}

MAP, mean arterial blood pressure; HR, heart rate; bpm, beats/min; Saline, rats were treated with saline for 1, 4 and 12 weeks; RSG, rats were treated with rosiglitazone for 1, 4 and 12 weeks. Values are expressed as means \pm SEM.

(A) Week 1

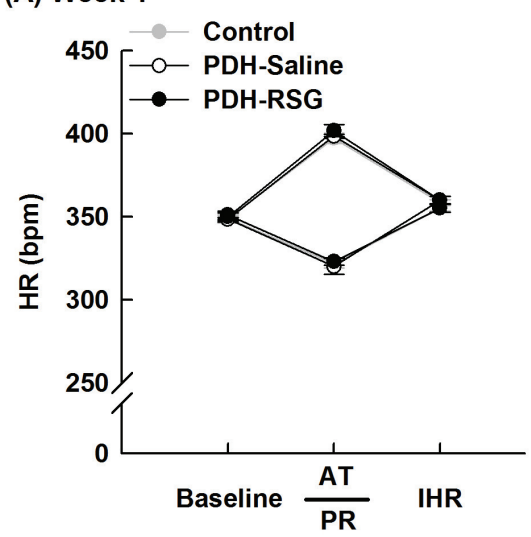

(B) Week 4



(C) Week 12

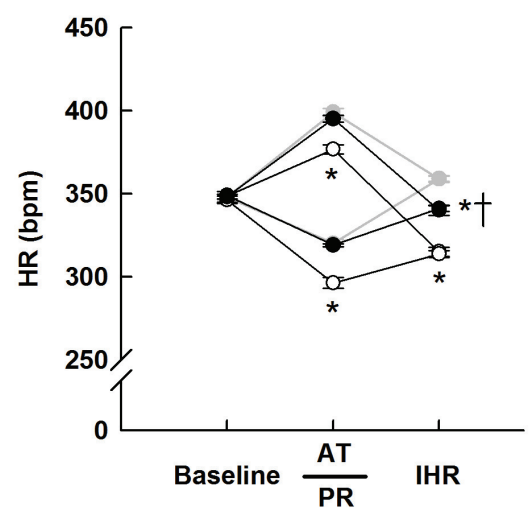

(D)

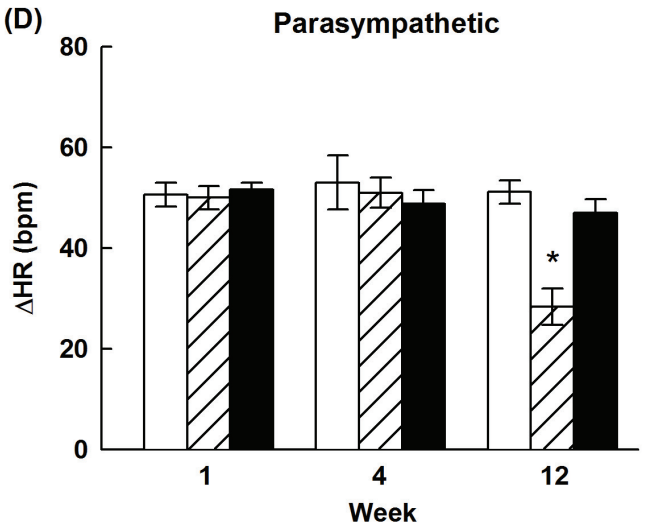

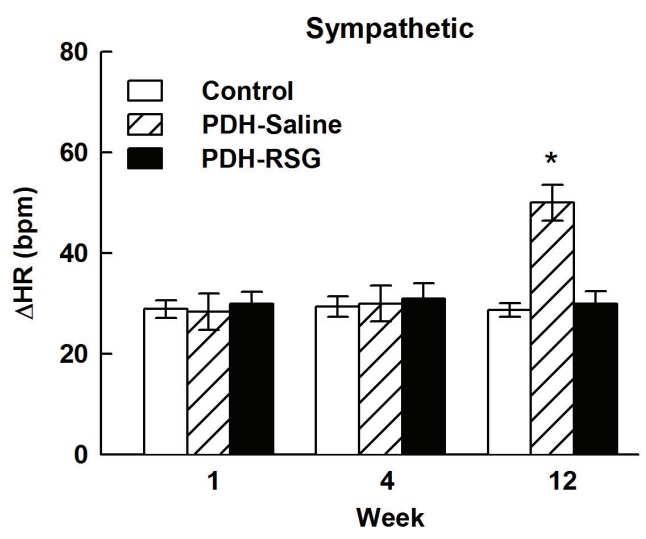

Fig. 2. $H R$ and cardiac autonomic responses in the saline- or RSG-treated PDH and control rats. (A, B, C) The baseline HR, HR responses after AT (the upper part of the diamond loop) or PR (the lower part of the diamond loop) treatment, and IHR at the end of 1 , 4 and 12 weeks. (D) Cardiac parasympathetic and sympathetic influences at the end of 1,4 and 12 weeks. $n=12$ per each group. $* P<0.05$ vs. the corresponding values of control rats; $+P<0.05$ vs. the corresponding values of the saline-treated PDH rats. Values are expressed as mean \pm SEM.

The IHR obtained after methylatropine plus propranolol treatments in the PDH rats with or without RSG treatment was not different from that of the timematched control rats at week 1 (Fig. 2A). The IHR of saline-treated PDH rats was lower at week 4 (Fig. 2B) and showed an even greater reduction at week 12 (Fig. 2C). RSG treatment for 4 weeks was unable to prevent the decreases in IHR. However, 12 weeks of RSG treatment significantly improved the lowered IHR in the PDH rats, although it was not completely restored. 
The cardiac sympathetic and parasympathetic influences in the PDH rats with or without RSG treatment were not different from those of the time-matched control rats at weeks 1 and 4 (Fig. 2D). As prediabetic hyperglycemia advanced into week 12, the cardiac parasympathetic influences were attenuated, and cardiac sympathetic influences were enhanced in the salinetreated PDH rats. With 12 weeks of RSG treatment, these cardiac autonomic functional changes in the $\mathrm{PDH}$ rats were able to be completely reversed.

\section{$B R S$}

Figures 3A-C show the representative plots of HR changes versus the MAP changes in response to various doses of $\mathrm{PE}$ and NP at the baseline in the salineor RSG-treated PDH and control rats at weeks 1, 4 and 12. At the end of week 1, the PE- and NP-BRS were not different between the saline-treated PDH and the timematched control rats (Fig. 3A). At week 4, the PE-BRS began to increase (more negative, $-3.89 \pm 0.05$ as compared to $-3.36 \pm 0.06 \mathrm{bpm} / \mathrm{mmHg}$ in the control rats, $P<0.05$ ), while the NP-BRS remained unchanged in the saline-treated $\mathrm{PDH}$ rats (Fig. 3B). As the prediabetic hyperglycemia advanced into week 12 , the enhancement in PE-BRS was more significant and the NP-BRS became attenuated in the saline-treated $\mathrm{PDH}$ rats (Fig. 3C and D). The enhanced PE-BRS was markedly eliminated by methylatropine treatment, but remained enhanced after propranolol treatment (Fig. 3D, PE-BRS). On the other hand, attenuation of NP-BRS was still observed after methylatropine treatment, but it was completely abolished by propranolol treatment (Fig. 3D, NP-BRS).

Four weeks of RSG treatment did not improve the enhanced PE-BRS in the PDH rats (Fig. 3B). However, 12 weeks of RSG treatment completely restored the enhanced PE-BRS and attenuated NP-BRS back to control levels (Fig. 3D).
(A) Week 1

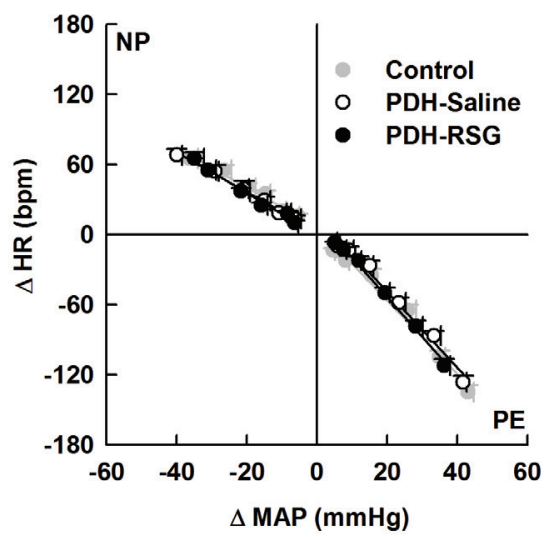

(B) Week 4

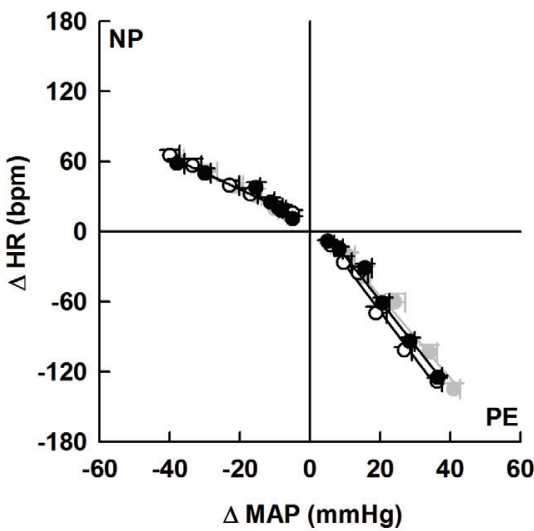

(C) Week 12



(D) Week 12


Fig. 3. PE-BRS and NP-BRS in the saline- or RSG-treated PDH and control rats. (A, B, C) The overall view of the MAP-HR relationship in response to various doses of PE and NP at baseline in the saline- or RSG-treated PDH and control rats at the end of 1, 4, or 12 weeks. (D) The PE- and NP-BRS at baseline and after AT or PR treatment in the saline- or RSG-treated PDH and control rats at the end of week 12. $n=12$ per group. $* P<0.05$ vs. the corresponding values of control rats. Values are expressed as mean $\pm \mathrm{SEM}$. 
(A) Week 1 -. . PDH-Saline


(B) Week 4


(C) Week 12




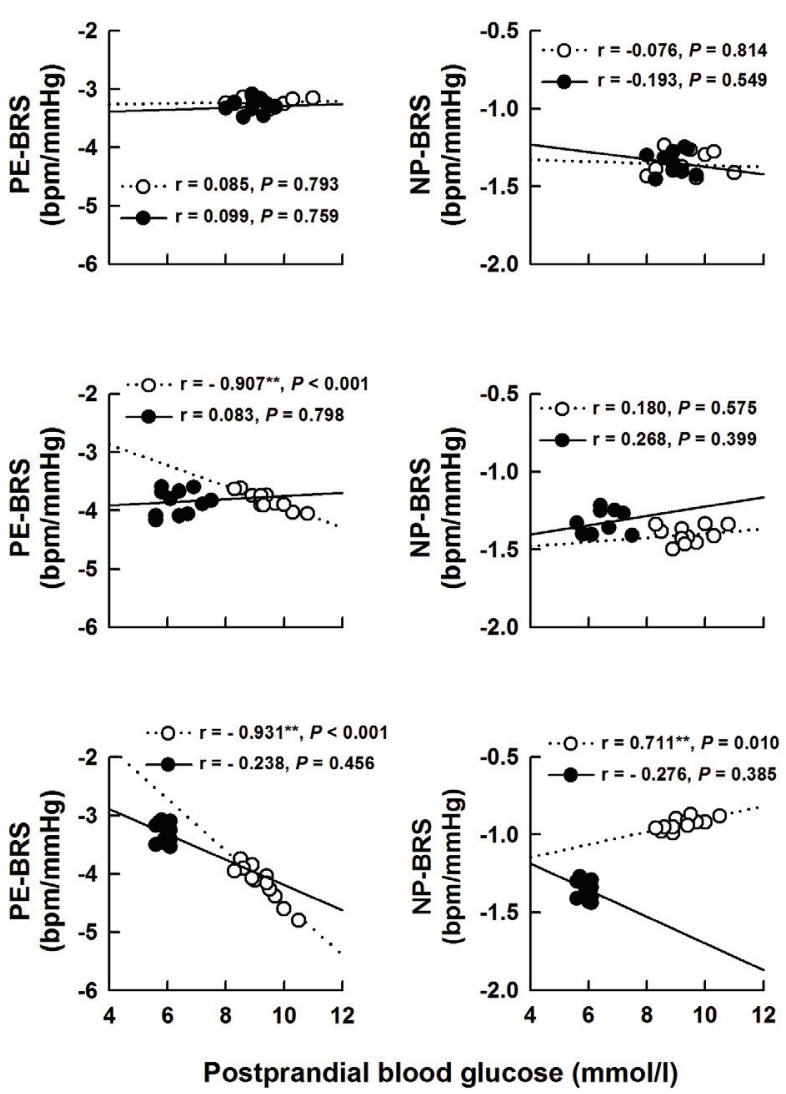

Fig. 4. Correlations of PE-BRS and NP-BRS with fasting and postprandial blood glucose levels in PDH rats. (A, B, C) The data include PDH rats treated with RSG or saline for 1 (upper panel), 4 (middle panel), and 12 (lower panel) weeks. $n=12$ per group. ** correlation is significant at the 0.01 level (2-tailed)

Correlations of PE-BRS and NP-BRS with fasting and postprandial blood glucose levels

As shown in Figure 4, in the saline-treated PDH rats, the PE-BRS was negatively correlated with the fasting and postprandial blood glucose levels starting after week 4 and was sustained up to week 12. The NPBRS became positively correlated with the fasting and postprandial blood glucose level only after week 12 . Interestingly, no correlations were found after RSG treatment.

\section{Discussion}

The present study demonstrated that elevations in blood glucose levels in the early stage (4 weeks) of prediabetes could lead to a significant change in PE-BRS in rats, even when the baseline MAP, HR, NP-BRS were unchanged. As prediabetic hyperglycemia progressed for a longer period (12 weeks), both PE-BRS and NP-BRS were abnormal. Four weeks of RSG treatment in rats with prediabetic hyperglycemia normalized blood glucose levels but did not improve PE-BRS. Twelve weeks of RSG treatment could completely restore both the PEBRS and NP-BRS. The correlation analysis revealed that the abnormalities in BRS seemed to be closely associated with the duration and severity of prediabetic hyperglycemia. The correlations, however, disappeared after chronic RSG treatment. Taken together, these results suggest a crucial role of elevated blood glucose in the development of BRS abnormalities, even moderate hyperglycemia as in the prediabetic state. Also, chronic RSG treatment in the prediabetic state might have favorable effects on BRS, in addition to its antihyperglycemic action.

The BRS, an important factor for detecting early abnormal cardiac autonomic function, is well-known to be impaired in-prediabetes (Iellamo et al. 2006) and diabetes (Dalla-Pozza et al. 2007, Weston et al. 1998). Blood glucose level by itself appeared to be a prominent factor in the development of diabetic autonomic dysfunction. It was demonstrated that impaired cardiac autonomic function was associated with impaired glucose 
tolerance (Wu et al. 2007) and with abnormal fasting glucose in non-diabetic human subjects (Stein et al. 2007). Moreover, in healthy subjects, it was shown that BRS was significantly reduced in response to acute hyperglycemia (Marfella et al. 2001), and BRS was inversely correlated with blood glucose levels independent of other factors (Lefrandt et al. 2000). These studies indicated that impaired glycemic control might account for the alteration of cardiac autonomic function. The present study showed that BRS abnormality developed after 4 weeks of prediabetic hyperglycemia in rats, and it was greater at week 12 (Fig. 3). As indicated in the correlation analysis (Fig. 4), the duration of prediabetic hyperglycemia might account for the severity of BRS abnormality in the PDH rats. Our results were consistent with those of previous studies and clearly demonstrated that elevated blood glucose levels, even in the prediabetic state, could be a contributing factor in the abnormal BRS.

The data from studies with pharmacological autonomic blockade in the 12-week PDH rats further revealed that the enhanced PE-BRS might have primarily involved abnormal parasympathetic responses in reflex, whereas the depressed NP-BRS was likely due to a faulty sympathetic function (Fig. 3D). In addition, the resting cardiac parasympathetic and sympathetic influences were found to be significantly attenuated and enhanced, respectively, in the 12-week PDH rats (Fig. 2D). Thus, the existing lower resting parasympathetic activity, which would allow easier further activation in response to hypertensive stimulation (PE), might have accounted for the enhancement of PE-BRS. In contrast, the higher existing resting sympathetic activity would make activation in response to the NP-induced hypotension more difficult, which might result in an attenuated NPBRS (Levy 1990). Nonetheless, the impaired BRS was associated with the abnormal parasympathetic component of baroreflex pathway (Weston et al. 1998) or diabetic duration (Dalla-Pozza et al. 2007) in type 1 diabetic patients. In the diabetic animals, baroreflex-mediated tachycardia (as in NP-BRS) was found to be attenuated (Maeda et al. 1995), but baroreflex-mediated bradycardia (as in PE-BRS) was either reduced (do Carmo et al. 2008) or augmented (Liu et al. 2008). The discrepancies in these observations, as well as with our data, could be related to the severity of hyperglycemia, differences in diabetic duration, or the methods of BRS analysis.

In the PDH rats, the baseline HR remained normal throughout the experiment, regardless of the existing enhanced cardiac sympathetic and attenuated parasympathetic influences and lower IHR at week 12 (Fig. 2C and D). The lower IHR might reflect the changes in electrophysiological properties of the sino-atrial node resulting from the imbalance of cardiac autonomic function (Machado and Brody 1989). Therefore, the progression of sympathetic stimulation and parasympathetic withdrawal on the heart could lead to a further decrease in IHR which would subsequently normalize the HR, as also seen in the 12-week PDH rats. In the PDH rats at weeks 1 and 4, the cardiac autonomic influences were normal. The IHR was normal at week 1 and was significantly lower at week 4 . However, the depression of IHR at week 4 was less than that of the 12week $\mathrm{PDH}$ rats (Fig. $2 \mathrm{~B}$ and $\mathrm{C}$ ). These results suggest that prediabetic hyperglycemia, unlike severe diabetic hyperglycemia (Maeda et al. 1995), might require a sufficient period of time (12 weeks) to effectively alter cardiac autonomic functions. Moreover, since the blood pressure was also unchanged in the PDH rats throughout experiments, the results of the present study further indicate that in the prediabetic state, subtle cardiac autonomic function changes (i.e., BRS) might occur prior to the changes in HR and blood pressure.

RSG treatment for 4 weeks could normalize blood glucose levels but did not effectively improve the altered BRS in the PDH rats (Fig. 3B). However, RSG treatment for 12 weeks could completely restore the BRS and cardiac autonomic functions to the control levels (Fig. 3D). Similarly, our previous study in normal rats has shown that 4 weeks of RSG treatment did not change BRS, however, the enhanced PE-BRS and attenuated NPBRS was found after 12 weeks of RSG treatment (Hsieh and Hong 2008). Although the effect of RSG on BRS in normal and diabetic rats remains controversial, these results suggest that a longer period of time ( $>4$ weeks) was required to allow RSG to exert its effect on cardiac autonomic control of HR (i.e., the BRS). In addition, the close correlations between blood glucose and BRS disappeared after RSG treatment (Fig. 4). These results imply that in addition to the anti-hyperglycemic effect, chronic RSG treatment may have further beneficial effects on baroreflex function.

The mechanisms by which RSG improves BRS under hyperglycemia are not fully understood. Reduction in circulatory NO bioavailability complicated by the enhanced oxidative stress has been suggested to be the contributing factor in cardiovascular (Giugliano et al. 1997) and baroreflex (Marfella et al. 1995) dysfunction 
with hyperglycemia. In addition to the well-known insulin sensitizing property that reduces blood glucose (Yki-Jarvinen 2004), RSG also has been shown to offer beneficial effects on antioxidant effects (Manning et al. 2008, Wiggin et al. 2008) and NO bioavailability (Bagi et al. 2004, Wang et al. 2007). Our previous study also demonstrated that 12 weeks of RSG treatment could increase endogenous NO production which was thought to contribute to the alteration of BRS in normal rats (Hsieh and Hong 2008). Thus, the ability of RSG to increase NO availability or to reduce oxidative stress may be related to the improvement of BRS in the $\mathrm{PDH}$ rats with chronic RSG treatment.

Recently, clinical trials and meta-analyses of clinical studies suggested that cautions need to be taken before RSG treatment of diabetic patients who have already had multiple cardiovascular autonomic neuropathies (Kaul et al. 2010). Despite the limitations in the present study, our data demonstrated that RSG might be of benefit in preserving the baroreflex function in prediabetic hyperglycemic rats.

In conclusion, the present study demonstrates that BRS was abnormal early during prediabetic hyperglycemia. In addition to its anti-hyperglycemic effects, chronic RSG treatment in prediabetic state could improve BRS and cardiac autonomic influences. The results of the present study may be of pharmacological importance for RSG in modifying cardiac autonomic function, in addition to its well-known effects on lipid and glucose metabolism.

\section{Conflict of Interest}

There is no conflict of interest.

\section{Acknowledgements}

The authors declare no conflict of interest. The authors would like to thank Dr. Wayne Huey-Herng Sheu for invaluable comments regarding this study. This study was supported by the grants of Taichung Veterans General Hospital (TCVGH-947308B) and (TCVGH-PU988101).

\section{References}

BAGI Z, KOLLER A, KALEY G: PPARgamma activation, by reducing oxidative stress, increases NO bioavailability in coronary arterioles of mice with Type 2 diabetes. Am J Physiol 286: H742-H748, 2004.

CHANG KS, LUND DD: Alterations in the baroreceptor reflex control of heart rate in streptozotocin diabetic rats. J Mol Cell Cardiol 18: 617-624, 1986.

DALLA-POZZA R, BECHTOLD S, BONFIG W, PUTZKER S, KOZLIK-FELDMANN R, SCHWARZ HP, NETZ H: Impaired short-term blood pressure regulation and autonomic dysbalance in children with type 1 diabetes mellitus. Diabetologia 50: 2417-2423, 2007.

DO CARMO JM, HALL JE, DA SILVA AA: Chronic central leptin infusion restores cardiac sympathetic-vagal balance and baroreflex sensitivity in diabetic rats. Am J Physiol 295: H1974-H1981, 2008.

GENUTH S, ALBERTI KG, BENNETT P, BUSE J, DEFRONZO R, KAHN R, KITZMILLER J, KNOWLER WC, LEBOVITZ H, LERNMARK A, NATHAN D, PALMER J, RIZZA R, SAUDEK C, SHAW J, STEFFES M, STERN M, TUOMILEHTO J, ZIMMET P; EXPERT COMMITTEE ON THE DIAGNOSIS AND CLASSIFICATION OF DIABETES MELLITUS: Follow-up report on the diagnosis of diabetes mellitus. Diabetes Care 26: 3160-3167, 2003.

GIUGLIANO D, MARFELLA R, COPPOLA L, VERRAZZO G, ACAMPORA R, GIUNTA R, NAPPO F, LUCARELLI C, D'ONOFRIO F: Vascular effects of acute hyperglycemia in humans are reversed by Larginine. Evidence for reduced availability of nitric oxide during hyperglycemia. Circulation 95: 1783-1790, 1997.

HSIEH PS, HONG LZ: Augmentation of nitric oxide is crucial for the time-dependent effects of rosiglitazone on blood pressure and baroreflex function in rats. J Hypertens 26: 83-92, 2008.

IELLAMO F, TESAURO M, RIZZA S, AQUILANI S, CARDILLO C, IANTORNO M, TURRIZIANI M, LAURO R: Concomitant impairment in endothelial function and neural cardiovascular regulation in offspring of type 2 diabetic subjects. Hypertension 48: 418-423, 2006.

KAUL S, BOLGER AF, HERRINGTON D, GIUGLIANO RP, ECKEL RH: Thiazolidinedione drugs and cardiovascular risks: a science advisory from the American Heart Association and American College of Cardiology Foundation. J Am Coll Cardiol 55: 1885-1894, 2010. 
KELLY AS, BANK AJ: The cardiovascular effects of the thiazolidinediones: a review of the clinical data. $J$ Diabetes Complications 21: 326-334, 2007.

LEFRANDT JD, MULDER MC, BOSMA E, SMIT AJ, HOOGENBERG K: Inverse relationship between blood glucose and autonomic function in healthy subjects. Diabetes Care 23: 1862-1864, 2000.

LEVY MN: Autonomic interactions in cardiac control. Ann N Y Acad Sci 601: 209-221, 1990.

LIU IM, CHANG CK, JUANG SW, KOU DH, TONG YC, CHENG KC, CHENG JT: Role of hyperglycaemia in the pathogenesis of hypotension observed in type-1 diabetic rats. Int J Exp Pathol 89: 292-300, 2008.

MACHADO BH, BRODY MJ: Contribution of neurogenic mechanisms to control of intrinsic heart rate. Am J Physiol 256: R231-R235, 1989.

MAEDA CY, FERNANDES TG, TIMM HB, IRIGOYEN MC: Autonomic dysfunction in short-term experimental diabetes. Hypertension 26: 1100-1104, 1995.

MANNING PJ, SUTHERLAND WH, WALKER RJ, WILLIAMS SM, DE JONG SA, BERRY EA: The effect of rosiglitazone on oxidative stress and insulin resistance in overweight individuals. Diabetes Res Clin Pract 81: 209-215, 2008.

MARFELLA R, VERRAZZO G, ACAMPORA R, LA MC, GIUNTA R, LUCARELLI C, PAOLISSO G, CERIELLO A, GIUGLIANO D: Glutathione reverses systemic hemodynamic changes induced by acute hyperglycemia in healthy subjects. Am J Physiol 268: E1167-E1173, 1995.

MARFELLA R, NAPPO F, MARFELLA MA, GIUGLIANO D: Acute hyperglycemia and autonomic function. Diabetes Care 24: 2016-2017, 2001.

MASIELLO P, BROCA C, GROSS R, ROYE M, MANTEGHETTI M, HILLAIRE-BUYS D, NOVELLI M, RIBES G: Experimental NIDDM: development of a new model in adult rats administered streptozotocin and nicotinamide. Diabetes 47: 224-229, 1998.

NOVELLI M, FABREGAT ME, FERNANDEZ-ALVAREZ J, GOMIS R, MASIELLO P: Metabolic and functional studies on isolated islets in a new rat model of type 2 diabetes. Mol Cell Endocrinol 175: 57-66, 2001.

PARRA RS, MENDES LA, FAZAN RJ, SALGADO HC: Pressure response to carotid occlusion in diabetic rats: effect of insulin therapy. Diabetes Res Clin Pract 68: 12-17, 2005.

STEIN PK, BARZILAY JI, DOMITROVICH PP, CHAVES PM, GOTTDIENER JS, HECKBERT SR, KRONMAL RA: The relationship of heart rate and heart rate variability to non-diabetic fasting glucose levels and the metabolic syndrome: the Cardiovascular Health Study. Diabet Med 24: 855-863, 2007.

THE DIABETES CONTROL AND COMPLICATIONS TRIAL RESEARCH GROUP: The effect of intensive diabetes therapy on measures of autonomic nervous system function in the Diabetes Control and Complications Trial (DCCT). Diabetologia 41: 416-423, 1998.

VINIK AI, MASER RE, MITCHELL BD, FREEMAN R: Diabetic autonomic neuropathy. Diabetes Care 26: 15531579, 2003.

WANG S, JIANG JL, HU CP, ZHANG XJ, YANG DL, LI YJ: Relationship between protective effects of rosiglitazone on endothelium and endogenous nitric oxide synthase inhibitor in streptozotocin-induced diabetic rats and cultured endothelial cells. Diabetes Metab Res Rev 23: 157-164, 2007.

WESTON PJ, JAMES MA, PANERAI RB, MCNALLY PG, POTTER JF, THURSTON H: Evidence of defective cardiovascular regulation in insulin-dependent diabetic patients without clinical autonomic dysfunction. Diabetes Res Clin Pract 42: 141-148, 1998.

WIGGIN TD, KRETZLER M, PENNATHUR S, SULLIVAN KA, BROSIUS FC, FELDMAN EL: Rosiglitazone treatment reduces diabetic neuropathy in streptozotocin-treated DBA/2J mice. Endocrinology 149: 4928-4937, 2008.

WU JS, YANG YC, LIN TS, HUANG YH, CHEN JJ, LU FH, WU CH, CHANG CJ: Epidemiological evidence of altered cardiac autonomic function in subjects with impaired glucose tolerance but not isolated impaired fasting glucose. J Clin Endocrinol Metab 92: 3885-3889, 2007.

YKI-JARVINEN H: Thiazolidinediones. N Engl J Med 351: 1106-1118, 2004. 